\title{
Uso da Internet como um Ambiente para Discussão de Casos Clínicos
}

\section{The Internet as an Environment for discussing Clinical Cases}

Silvia Itzcovici Abensur ${ }^{1}$

Hugo Abensur ${ }^{2}$

Denise Maria Avancini Costa Malheiros ${ }^{1}$

Roberto Zatz ${ }^{1}$

Rui Toledo Barros ${ }^{2}$

PALAVRAS-CHAVE:

- Diagnóstico;

- Estudos de Casos;

- Internet;

- Biópsia;

- Nefrologia;

- Educação Continuada.

KEY-WORDS:

- Diagnosis;

- Case Reports;

- Internet;

- Biopsy;

- Nephrology

- Education, Continuimg.

Recebido em: 17/07/2006

Reencaminhado em: 07/12/2006

Aprovado em: 08/05/2007

\section{A B S T R A C T}

An environment was built on the Internet for discussing clinical cases on the website of the Brazilian Society of Nephrology (http://www.sbn.org.br). The aim of this work is to present the operation of this system and the improvements made since its introduction in September 2001. Up to the present moment, 62 cases have been published and diagnosed. All of them are stored in the website for consultation without any previous registration. The Internet also proved to be a promising environment for clinical-pathological discussion for allowing the participation of professionals from distant centers and without local conditions for this kind of activity of great importance for continuing education in Medicine. 


\section{INTRODUÇÃO}

O pioneiro do método de estudo de casos (case method) foi Christopher Columbus Langdell, que, ainda durante sua graduação na Harvard Law School, de 1851 a 1854, desenvolveu uma enciclopédia de dados judiciais. Em 1870, assumiu a direção da escola e imediatamente implantou o método de casos, no qual os alunos liam somente as fontes originais de cada caso, discutiam, analisavam e apresentavam suas próprias conclusões e interpretações. A utilização produtiva do método era garantida pela introdução do formato de perguntas e respostas, o método socrático, salientando fatos do caso, raciocínio judicial, princípios e teorias, e comparações com outros casos ${ }^{1}$.

Na Harvard Business School, fundada em 1908, este método só foi implantado em 1920, na posse do segundo diretor, Wallace P. Donham, graduado na Harvard Law School. A idéia foi adotada por outras instituições.

No caso da educação médica, o método foi proposto não por um professor, mas pelo aluno Walter Bradford Cannon há cem anos ${ }^{2}$. Durante o curso de graduação na Harvard Medical School, Cannon teve um companheiro de quarto, Harry Bigelow, estudante da Harvard Law School, que o deixou muito impressionado pelo entusiasmo dos alunos de Direito com o método de ensino de $\operatorname{casos}^{2-4}$. Cannon, com seu entusiasmo, persuadiu seu instrutor em neurologia, G. L. Walton, a realizar um experimento em dezembro de 1899, usando a história de um de seus pacientes particulares, que resultou na publicação de um artigo sobre o método do caso no ensino da Medicina ${ }^{5}$.

Depois que Cannon introduziu a idéia do ensino com o estudo de casos na educação médica, Richard Clarke Cabot tornou-se o mais ardente defensor deste método, utilizandoo, a partir de 1900, como parte do treinamento dos alunos do terceiro ano da Harvard Medical School.

Devido à reconhecida habilidade diagnóstica do Cabot na Harvard Medical School e no Massachusetts General Hospital, em Boston, Massachusetts, a elaboração do pensamento diagnóstico tornou-se mais "científica". Em 1896, ele escreveu um guia para exame de sangue, seguido, em 1905, por um livro-texto de diagnóstico que foi padrão por muitos anos ${ }^{6}$. Cabot dedicou-se bastante à pesquisa clínica, tendo reunido numerosas informações sobre os casos sob seu comando ${ }^{2}$.

Em 1910, com a ajuda do então fisiologista Walter Cannon, Cabot desenvolveu a conferência clínico-patológica (ClinicalPathological Conference), como resultado de sua ênfase no estudo de casos e na confirmação do diagnóstico pela autópsia7.

Suas conferências eram regularmente realizadas no Massachusetts General Hospital, e a partir de 1924 os registros dessas conferências começaram a ser publicados no Boston Medical and Surgical Journal. Assim, este exercício ficou associado ao nome de Cabot, como uma significativa contribuição à medicina americana ${ }^{8}$. Mais tarde, esse jornal tornou-se o New England Journal of Medicine.

Em 2000, celebrando o novo milênio, o editor Robert E. Scully, do New England Journal of Medicine, dedicou um prefácio ao papel de Cabot, fundador das conferências clínicopatológicas que continuam a aparecer nesse jornal, tendo atingido a marca de mais de 5 mil casos na época?

É muito importante aprender os passos que se devem seguir para avaliar um paciente doente. Na seqüência, incluem a queixa, a história da moléstia atual, os antecedentes e o exame físico, que são detalhes que auxiliam na análise e conhecimento da doença e na determinação do possível diagnóstico.

A importância da discussão de casos clínicos na educação médica está na conexão entre a ciência básica e os problemas reais da medicina prática.

A discussão anatomoclínica é um importante instrumento de aprendizado em Medicina empregado durante a formação do profissional, mas nem todos têm a oportunidade de participar na atividade profissional. Foi criado, então, um espaço virtual como mais uma alternativa para este tipo de discussão na área de Nefrologia no website da Sociedade Brasileira de Nefrologia (SBN) ${ }^{10}$.

\section{FUNCIONAMENTO}

Mensalmente, um novo caso clínico é disponibilizado. Na página inicial aparece uma lista com várias opções. O link "Introdução" apresenta o nome do relator e da equipe responsável e a identificação do caso, mantendo-se o sigilo sobre a identidade do paciente. No link "Apresentação" aparece uma tela com a História da Moléstia Atual e os Antecedentes, acompanhados da documentação clínicolaboratorial pertinente (exames de laboratório, imagens, etc.). No momento da introdução do caso, o link "Diagnóstico" apresenta cinco opções de diagnóstico. Todos os usuários podem registrar seu palpite diagnóstico anonimamente, e, após a escolha, aparece uma tabela com os resultados, em porcentagens, dos palpites acumulados para cada alternativa de diagnóstico do respectivo caso.

Normalmente, o diagnóstico correspondente é divulgado apenas após duas semanas de exposição do caso clínico no website. Durante este período, os usuários podem também participar da discussão das implicações fisiopatológicas no item "Mural" da lista de opções, registrando questões ou 
comentários que são prontamente respondidos pelos respectivos relatores responsáveis.

No momento da divulgação do diagnóstico, o link "Diagnóstico" apresenta o resultado da biópsia com suas respectivas ilustrações (por exemplo, microscopia óptica e imunofluorescência), a conclusão diagnóstica e a discussão.

A listagem de todos os casos já elucidados e suas respectivas informações e discussões foram organizadas no site, permitindo o rápido e fácil acesso aos conteúdos pelo usuário. As páginas estáticas deste espaço de discussão foram criadas na linguagem HTML (HyperText Mark-Up Language), e as páginas dinâmicas (mecanismo de registro e visualização dos comentários arquivados do Mural e mecanismo de registro dos palpites diagnósticos) foram elaboradas em ASP (Active Server Pages).
O banco de dados com os registros dos comentários (nome, $e^{-}$ mail, instituição/cidade e comentário) e dos palpites diagnósticos foi construído em Access Microsoft ${ }^{\circledR}$.

\section{RESULTADOS}

Este sistema está ativo desde setembro de 2001 e atualmente foi divulgado o diagnóstico do Caso Clínico número 62. Todos os casos já divulgados e diagnosticados estão arquivados no site para consulta por qualquer interessado sem a necessidade de registro prévio ${ }^{10}$. A seguir, serão relatados alguns resultados coletados no site em 07/ 12/2006. Os 62 casos arquivados são provenientes de oito instituições, conforme a Tabela 1.

TABELA 1

Distribuição dos 62 casos arquivados pelas oito instituições participantes

\begin{tabular}{l|c|c}
\hline \multirow{2}{*}{ Instituição } & \multicolumn{2}{|c}{ Casos } \\
\cline { 2 - 3 } & Quantidade & $\%$ \\
\hline Hospital das Clínicas da Faculdade de Medicina da USP & 53 & 85,5 \\
\hline Faculdade de Medicina de Ribeirão Preto da USP & 2 & 3,2 \\
\hline Faculdade de Ciências Médicas da Santa Casa de São Paulo & 2 & 3,2 \\
\hline Universidade Federal de São Paulo & 1 & 1,6 \\
\hline Hospital Beneficência Portuguesa de São Paulo & 1 & 1,6 \\
\hline Faculdade de Medicina do ABC & 1 & 1,6 \\
\hline Faculdade de Medicina de São José do Rio Preto & 1 & 1,6 \\
\hline FM Botucatu - Unesp & 1 & 1,6 \\
\hline
\end{tabular}

O mecanismo de registro dos palpites diagnósticos foi implantado a partir do Caso 3. Do levantamento realizado na distribuição dos palpites diagnósticos arquivados, observouse que em somente 34 dos 60 casos clínicos (56,7\%) a alternativa mais votada foi o diagnóstico correto. Em termos de palpites acumulados nos diversos casos, 4.750 do total de 13.208 palpites - ou seja, 36\% - correspondem ao diagnóstico correto dos respectivos casos.

O "Mural" foi introduzido no espaço a partir do Caso 9 e contém atualmente 489 comentários, variando de 0 a 21 comentários por caso, o que inclui tanto os comentários dos usuários interessados, como as respectivas respostas fornecidas pelos relatores responsáveis, resultando numa média de 9,2 comentários por caso.

Pelos comentários registrados, tem-se notado a participação de usuários de diversas regiões do Brasil. Durante todo o período de exposição do Caso 61, isto é, desde a sua introdução até a data da introdução do Caso 62, registraram- se 2.096 usuários que visitam sempre o site, mas que, por certa inibição, nem sempre participam da discussão do Mural. Foi criado o link "Cadastre-se", a partir do Caso 6, para que os usuários registrem seu interesse no aviso de novidades por e-mail, e atualmente têm-se 1.041 usuários cadastrados.

Uma forma de enriquecer o conteúdo desta biblioteca virtual foi a inclusão do Material Didático de Apoio, a partir do Caso 30. Geralmente, são preparados textos ilustrados e animados ou apresentações em PowerPoint Microsoft ${ }^{\circledR}$ explicativas sobre o assunto, alguns com o apoio do Departamento de Fisiologia e Fisiopatologia da Sociedade Brasileira de Nefrologia (SBN). Esse material é disponibilizado juntamente com a divulgação do diagnóstico e serve de apoio ao estudo dos casos discutidos. Parte desse material aparece também em Fisiopatologia Clínica - volume 2 - Sistema Renal ${ }^{11}$. Cada material de apoio pode ser acessado diretamente pelo respectivo link na página do diagnóstico do caso clínico relacionado ou pela listagem completa dos materiais de apoio disponíveis. 
Outra mudança introduzida foi a forma de apresentar a discussão no momento da divulgação do diagnóstico. Assim, cada alternativa diagnóstica proposta na apresentação do caso é discutida, sendo enumeradas as razões para aceitá-la ou rejeitá-la, acompanhadas das respectivas referências bibliográficas.

O recurso de discutir, na divulgação do diagnóstico, cada alternativa proposta na apresentação do caso permite ensinar o modo de analisar e abordar um caso clínico e seus respectivos exames necessários para alcançar o diagnóstico e também mostra os diagnósticos diferenciais. Isto estimula e promove a construção do pensamento crítico e dedutivo, sendo menos importante destacar o diagnóstico correto do que apresentar uma análise lógica e instrutiva das condições pertinentes envolvidas ${ }^{3}$.

\section{DISCUSSÃO}

Outra forma de exibir a discussão anatomoclínica tem sido a publicação de casos clínicos em periódicos. Esta forma de discussão é restrita, pois questionamentos do leitor só poderiam ser respondidos pelo autor sem a participação de outros leitores, e é também pouco dinâmica.

A Faculdade de Medicina da USP desenvolveu uma tecnologia que interliga entidades, por meio de internet bandalarga ou videoconferência, e permite o acompanhamento online de aulas, autópsias, discussões, programas de prevenção e consultas à distância ${ }^{12}$. Assim, professores, estudantes e pacientes de distantes regiões do Brasil pertencentes a localidades habilitadas com senha podem recepcionar o sinal do site da USP13-15 e ter acesso à medicina de ponta sem viajar para os grandes centros urbanos. Antes da autópsia, os alunos debatem o caso clínico com especialistas. Esta tecnologia é restrita aos centros que estão interligados e aos participantes presentes no momento agendado para o evento.

O MedStudents ${ }^{16}$ é um site criado e mantido pelos acadêmicos da UFRJ que contém casos clínicos bem documentados, ativo desde maio de 2001. Já foram apresentados casos clínicos de diversas áreas da Medicina, como: Pediatria, Clínica Médica, Otorrinolaringologia e Medicina Interna. Antes da divulgação do diagnóstico do caso clínico, usuários registrados podem inserir comentários sobre o possível diagnóstico, mas não há debate com a participação dos elaboradores do caso clínico.

A vantagem do espaço criado para discussão descrito neste trabalho está na possibilidade de participação de qualquer usuário, sem cadastro prévio ou necessidade de tecnologia especial, durante um período mais extenso (15 a 20 dias). E qualquer questionamento do usuário é rapidamente respondido pelos relatores responsáveis e pode ser visualizado e comentado por todos os usuários.

\section{CONCLUSÃO}

Este espaço pode ser considerado uma opção de e-learning, que é definido pela Comissão Européia de e-Learning como o uso de novas tecnologias multimídia e da internet para melhorar a qualidade da aprendizagem mediante o acesso a recursos e serviços, e a colaborações e intercâmbios a grande distância' ${ }^{17}$.

A internet mostrou-se também um ambiente propício à discussão anatomoclínica, uma vez que permite a participação de profissionais de centros distantes e sem condição local para este tipo de atividade, tão importante para o contínuo aprendizado da Medicina.

\section{AGRADECIMENTO}

Os autores são gratos à diretoria da Sociedade Brasileira de Nefrologia (SBN), que cedeu espaço em sua homepage para hospedar a sessão de discussão de casos clínicos.

\section{REFERÊNCIAS}

1. Garvin DA. Making the case: professional education for the world of practice. Harv Mag. 2003; 105(1): 56-65.

2. Breathnach CS. Problems for students or for teachers? Med Educ. 2000; 34(12): 974-5.

3. Castleman B, Dudley HR. A brief history of the clinicopathological case series. [online]. [capturado $07 \mathrm{dez}$. 2006]. Disponível em: http:/ /www.mgh.harvard.edu/ pathology/cpc-history.htm.

4. Scully RE. Cambridge 02138: ACLU, Harvard architecture, personnel costs, eslamic democracy. Harv Mag. 2003; 105(2):4.

5. Cannon WB. The case method of Teaching Systematic Medicine. Boston Med Surg J. 1900; 142: 563-4.

6. Dodds TA. Richard Cabot: medical reformer during the progressive era (1890-1920). Ann Intern Med. 1993; 119(5):417-22.

7. Walker HK, Hall WD, Hurst JW, eds. Clinical methods: the history, physical and laboratory examinations. $3^{\text {rd }}$ ed. Boston: Butterworth; c1990.

8. Crenner C. Diagnosis and authority in the early-twentiethcentury medical practice of Richard C. Cabot. Bull Hist Med. 2002; 76(1): 30-55. 
9. Kantoff PW, Oliva E. Case 1-200: a 27-year-old man with a painful retroperitoneal mass. N Engl J Med. 2000; 342(2):115-22.

10. Sociedade Brasileira de Nefrologia. Discussão de Casos: Nefrologia/USP. [online]. [capturado 07 dez. 2006]. Disponível em: http://www.sbn.org.br.

11. Zatz R. Fisiopatologia clínica: v.2: sistema renal. [São Paulo]: Atheneu; 2001.

12. Estação Digital Médica. [online]. [capturado 07 dez. 2006]. Disponível em: http:/ / www.estacaodigitalmedica.com.br.

13. Experiência em tele-educação do PR levada a outros Estados. Jornal do CRM/PR, Paraná, 2005 abr./maio, n.67; p.15.

14. Videoconferência de telepatologia. Jornal do CRM-PR, Paraná, 2005 nov./dez., n.70; p.15.
15. Programa de telepatologia do CRM com oito edições em 2006. Jornal do CRM-PR, Paraná, 2006 jan./mar., n.71; p.16.

16. MedStudents. [online]. [capturado 07 dez. 2006]. Disponível em: http:/ / www.medstudents.com.br.

17. [Comissão Européia de E-Learning]. [online]. [capturado 07 dez. 2006]. Disponível em: http:/ / elearningeuropa.info.

\section{CONFLITO DE INTERESSE}

Declarou não haver.

\section{ENDEREÇO PARA CORRESPONDÊNCIA}

Silvia Itzcovici Abensur

Avenida Macuco, 58

04523-000 - São Paulo - SP

E-mail: sabensur@usp.br 